\title{
Surface analytical investigation on organometal triiodide perovskite
}

\author{
Chenggong Wang \\ Department of Physics and Astronomy, University of Rochester, Rochester, New York 14627 \\ Xiaoliang Liu \\ Hunan Key Laboratory for Super-Microstructure and Ultrafast Process, College of Physics and Electronics, \\ Central South University, Changsha, Hunan 410083, People's Republic of China \\ Congcong Wang \\ Department of Physics and Astronomy, University of Rochester, Rochester, New York 14627
}

Zhengguo Xiao, Cheng Bi, Yuchuan Shao, and Jinsong Huang

Department of Mechanical and Materials Engineering, University of Nebraska, Lincoln, Lincoln, Nebraska 68588

Yongli Gao ${ }^{\text {a) }}$

Department of Physics and Astronomy, University of Rochester, Rochester, New York 14627

(Received 12 December 2014; accepted 9 March 2015; published 20 March 2015)

In a little over a year, there has been an unexpected breakthrough and rapid evolution of highly efficient solid-state hybrid solar cells based on organometal trihalide perovskite materials. This technology has the potential to produce solar cells with the very highest efficiencies while retaining the very lowest cost. The authors have measured the electronic density of states of $\mathrm{CH}_{3} \mathrm{NH}_{3} \mathrm{PbI}_{3}$ using ultraviolet photoemission spectroscopy (UPS), inverse photoemission spectroscopy (IPES), and x-ray photoemission spectroscopy (XPS). The valence band maximum and conduction band minimum positions are obtained from the UPS and IPES spectra, respectively, by linear extrapolation of the leading edges. The authors investigate the Au/perovskite and $\mathrm{C}_{60}$ /perovskite interfaces by UPS and XPS. An interface dipole of $0.1 \mathrm{eV}$ is observed at $\mathrm{Au} /$ perovskite interface. The energy levels of perovskite shift upward by ca. $0.4 \mathrm{eV}$ with Au coverage of $64 \AA$ upon it, resulting in band bending, hence a built-in field in perovskite that encourages hole transport to the interface. The XPS results show a strong initial shift of core levels to lower binding energy in the perovskite, which indicates that electrons transfer from the perovskite film to fullerene molecules. Further deposition of fullerene forms $\mathrm{C}_{60}$ solid, accompanied by the reduction of the electron transfer. The strongest electron transfer happened at 1/4 monolayer of fullerene. (c) 2015 American Vacuum Society. [http://dx.doi.org/10.1116/1.4915499]

\section{INTRODUCTION}

Methylammonium lead trihalides have attracted great attention as candidates for the next generation solar cells as the power conversion efficiency (PCE) has exceeded $19 \% .{ }^{1-7}$ The advantages of these materials are solutionprocessable, low cost, of large carrier mobility and long carrier lifetime, and abundance in nature. ${ }^{8-13}$ The solutionprocessability makes it high possibility for low energy fabrication of large area photovoltaic platforms. Grätzel et al. ${ }^{14}$ brought the efficiency to $15 \%$ by depositing $\mathrm{PbI}_{2}$ on nanoporous $\mathrm{TiO}_{2}$ and subsequently submerging it into a $\mathrm{CH}_{3} \mathrm{NH}_{3} \mathrm{I}$ solution. Snaith's group demonstrated that a planar heterojunction perovskite solar cell by vapor deposition, without the mesoporous electrode in typical sensitized solar cells, could have the efficiency as high as 15\%., Zhou et al. ${ }^{16}$ recently reported the highest PCE as $19.3 \%$ by using polyethyleneimine ethoxylated modified indium tin oxide (ITO) and yttrium-doped $\mathrm{TiO}_{2}\left(\mathrm{Y}-\mathrm{TiO}_{2}\right)$ was used as the electron transportation layer. It is expected that the efficiency of $20 \%$ or higher can be reached by optimizing the

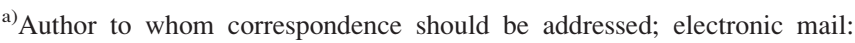
ygao@pas.rochester.edu
}

device structures. Au is most widely used as an electrode in solar cells based on organometal halide perovskite. It is believed that a typical planar structure of $\mathrm{Au} /$ perovskite and an electron transport layer such as $\mathrm{C}_{60}$ can used to build an effective solar cell. Shao et al. ${ }^{17}$ showed that the fullerene layers could effectively passivate the charge trap states at both $\mathrm{CH}_{3} \mathrm{NH}_{3} \mathrm{PbI}_{3}$ perovskite film surfaces and grain boundaries, as the fullerene eliminated the photocurrent hysteresis and doubled the efficiency of perovskite solar cells. It was also elucidated by Wang et al. ${ }^{18}$ that the charge trap density on perovskite surface was reduced by $10-100$ times, contributing to the record fill factor of above $80 \%$ in the planar heterojunction device. Therefore, it is imperative to investigate the interface formation and energetics of $\mathrm{Au} /$ perovskite and $\mathrm{C}_{60} /$ perovskite interfaces to better understand the underlining physical mechanisms.

We report our study using ultraviolet photoemission spectroscopy (UPS), inverse photoemission spectroscopy (IPES), and $\mathrm{x}$-ray photoemission spectroscopy (XPS) on Au/perovskite and $\mathrm{C}_{60} /$ perovskite interfaces. We measured directly the evolution of energy levels as $\mathrm{Au}$ or $\mathrm{C}_{60}$ was deposited successively layer by layer on a perovskite film. Au electrode efficiently lifted the energy levels of perovskite, and the valence band (VB) edge of perovskite was pulled up by 
$0.4 \mathrm{eV}$, from 1.0 to $0.6 \mathrm{eV}$. This indicated a weak energy loss as holes transfer from perovskite to Au. We monitored the changes of the valence electronic structures, including the highest occupied molecular orbital (HOMO), the lowest unoccupied molecular orbital (LUMO), and the vacuum level (VL), as $\mathrm{C}_{60}$ was successively deposited layer by layer on perovskite film coated on ITO substrate. We also monitored the core levels change by XPS. These investigations provide an important insight to the improvement of the perovskite solar cells by fullerene layers and the understanding of Au/perovskite-based organic solar cells.

\section{EXPERIMENT}

UPS measurements were performed using a modified VG ESCA Lab system, an ultrahigh vacuum (UHV) system equipped with a He discharge lamp. The UHV system consists of three interconnecting chambers, a spectrometer chamber, an in-situ oxygen plasma treatment chamber, and an evaporation chamber with a precision air leak valve. The base pressure of the spectrometer chamber is typically $8 \times 10^{-11}$ Torr. The base pressure of the evaporation chamber is typically $1 \times 10^{-6}$ Torr. The UPS spectra were recorded by using unfiltered $\mathrm{He} \mathrm{I}(21.2 \mathrm{eV})$ excitation as the excitation source with the sample biased at $-5.00 \mathrm{~V}$ to observe the low-energy secondary cutoff. The UV light spot size on the sample is about $1 \mathrm{~mm}$ in diameter. The XPS studies were performed with a $\mathrm{MgK}_{\alpha} \mathrm{x}$-ray source $(1253.6 \mathrm{eV})$. The typical instrumental resolution for UPS and XPS measurements are 0.2 and $1.4 \mathrm{eV}$, respectively. The IPES spectra were taken with a custom-made spectrometer composed of a commercial Kimball Physics ELG-2 electron gun and a band pass photon detector prepared according to an existing design. The combined resolution (electron + photon) of the IPES spectrometer was determined to be $\sim 0.6 \mathrm{eV}$ from the Fermi edge of an evaporated Au film. All depositions were done at room temperature and monitored with a quartz crystal microbalance. For the film fabrication, $\mathrm{PbI}_{2}$ and MAI were first dissolved in dimethylformamide and 2-propanol, respectively, as precursor solutions. The precursors were then spun onto poly(3,4-ethylenedioxythiophene) poly(styrenesulphonate) (PEDOT:PSS) covered ITO glass with a $\mathrm{PbI}_{2}$ layer underneath an MAI layer. A supersaturated hot solution of $\mathrm{PbI}_{2}$ was used for quick drying to obtain a pin-hole free and conformal $\mathrm{PbI}_{2}$ layer on the PEDOT:PSS surface. Since $\mathrm{PbI}_{2}$ has relatively low solubility in 2-propanol, the spin coating of MAI solution did not wash off the $\mathrm{PbI}_{2}$. The bilayer film was then annealed at a temperature of $100^{\circ} \mathrm{C}$ for $120 \mathrm{~min}$.

\section{RESULTS AND DISCUSSION}

Figure 1 is the XPS full scan spectrum of the perovskite film on top of an ITO/PEDOT:PSS substrate. The binding energy (BE) is referenced to the Fermi level. The sample displays carbon, nitrogen, iodine, and lead, and the surface composition $\mathrm{C}: \mathrm{N}: \mathrm{Pb}: \mathrm{I}=0.9: 0.8: 1: 2.4$ can be confirmed by the core level intensity, indicating a bit of deviation from the stoichiometry of $\mathrm{CH}_{3} \mathrm{NH}_{3} \mathrm{PbI}_{3}$. The relatively low

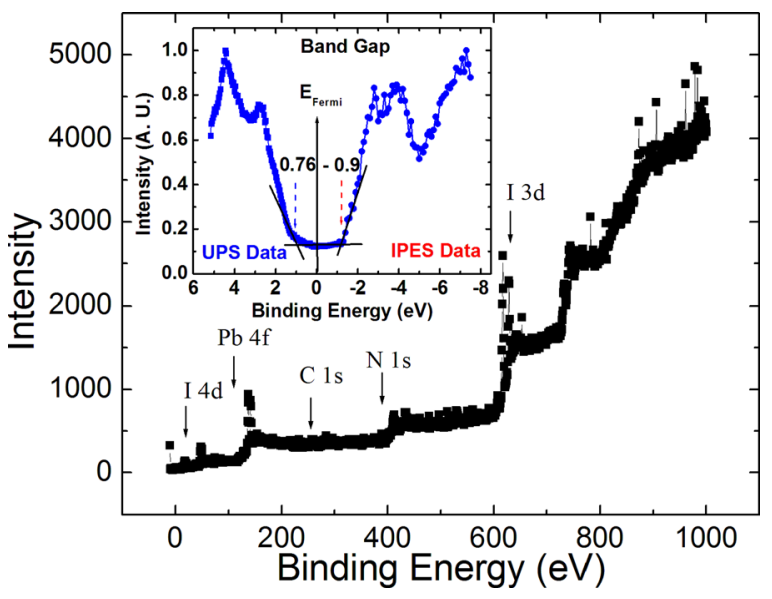

FIG. 1. (Color online) XPS full scan of the perovskite film. Surface composition $\mathrm{C}: \mathrm{N}: \mathrm{Pb}: \mathrm{I}=0.9: 0.8: 1: 2.4$. Inset: the band gap of the perovskite film.

proportions of nitrogen, carbon, and iodide may be due to the deficiency of $\mathrm{CH}_{3} \mathrm{NH}_{3} \mathrm{I}$. The $\mathrm{CH}_{3} \mathrm{NH}_{3} \mathrm{PbI}_{3}$ is not thermally stable at temperatures above $150{ }^{\circ} \mathrm{C}$ due to its low dissociation energy. ${ }^{19-21}$ Thermal annealing is a necessary step in the fabrication process of perovskite films in order to have enough interdiffusion between $\mathrm{CH}_{3} \mathrm{NH}_{3} \mathrm{I}$ and $\mathrm{PbI}_{2}$. However, thermal annealing may also dissociate partly $\mathrm{CH}_{3} \mathrm{NH}_{3} \mathrm{PbI}_{3}$ into $\mathrm{PbI}_{2}$ and $\mathrm{CH}_{3} \mathrm{NH}_{3} \mathrm{I}$, and the $\mathrm{CH}_{3} \mathrm{NH}_{3} \mathrm{I}$ may subsequently evaporate, resulting in reduced $\mathrm{N}$ content. In the inset, we show the UPS and IPES spectrum of the perovskite film. The origin in Fig. 1 inset is the Fermi level, which was calibrated by UPS and IPES on the Au/Si substrates. The spectra on the left side of the Fermi level are the valence bands from UPS while the spectra on the right side are the conduction bands from IPES. For the pristine perovskite film, the VB feature is very clear and the valence band maximum $(\mathrm{VBM})$ is $0.76 \mathrm{eV}$. The conduction band minimum (CBM) is $-0.90 \mathrm{eV}$, which are consist with previous reports. ${ }^{3,22}$ We define the onset as the extrapolation of the leading edge (closer to the Fermi level) in the spectrum. The results are an injection energy gap of about $1.66 \mathrm{eV}$ which is consistent with the previous report. ${ }^{22,23}$

In Fig. 2, the evolution of the UPS spectra is presented as a function of the thickness of Au deposited on the perovskite film. We normalized all the spectra to the same height for visual clarity. Figure 2(a) is the UPS data of the cut-off region and Fig. 2(b) is the HOMO region. The work function (WF) of perovskite film is measured to be $4.7 \mathrm{eV}$, and it decreases to $4.4 \mathrm{eV}$ upon the deposition of $0.5 \AA$ Au. With the subsequent deposition of $\mathrm{Au}$, the WF increases rapidly at first, and finally saturates at $5.2 \mathrm{eV}$ with Au coverage of $64 \AA$ A. Figure 2(b) presents the UPS data of the highest lying VB regions, in which the VBM of perovskite film displays as $1.0 \mathrm{eV}$. The $\mathrm{VB}$ edges are obtained using linear extrapolation as illustrated in our previous works. ${ }^{24,25}$ With Au deposition, a finite density of valence states are observed within the gap of $\mathrm{CH}_{3} \mathrm{NH}_{3} \mathrm{PbI}_{3}$. We observed clear Fermi surface when Au coverage reaches $8 \AA$ and a true metallic Fermi edge develops completely with the Au deposition of up to $64 \AA$. This effect is just similar to that confirmed by Dürr 


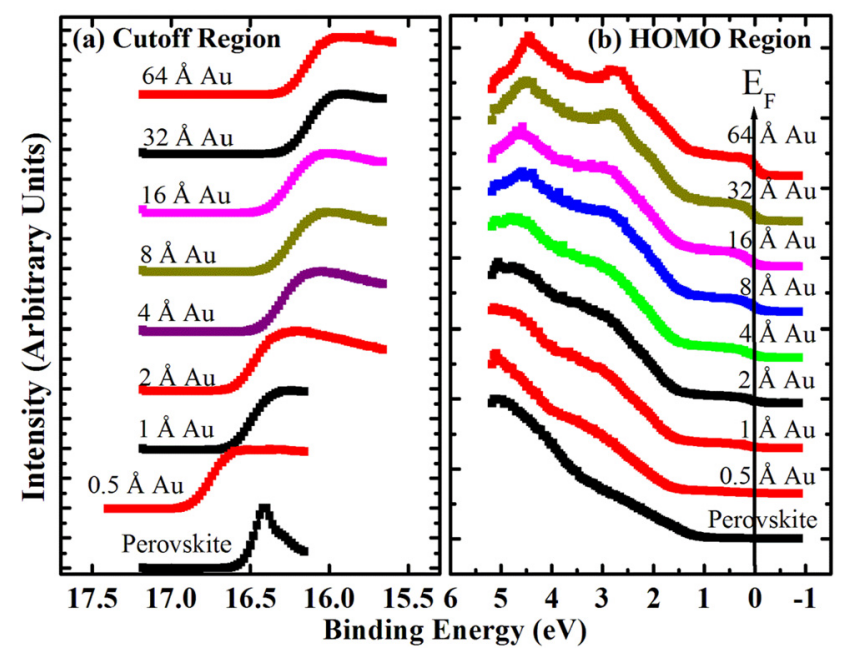

FIG. 2. (Color online) UPS data for Au/perovskite: (a) the cut-off region and (b) the HOMO region.

et $a .^{26}$ as $\mathrm{Au}$ was deposited on diindenoperylene. We also noticed a sharp shift of $0.3 \mathrm{eV}$ toward high $\mathrm{BE}$ at $0.5 \AA \mathrm{Au}$ deposition upon perovskite film, which can be ascribed to sample charging during UPS at lower Au coverage. As reported by Koch et al. ${ }^{27}$ and Oji et al., ${ }^{28}$ Au clusters formed on some organic films as Au was deposited upon them with lower Au over layer.

In Fig. 3, the evolution of UPS spectra due to different $\mathrm{C}_{60}$ thickness is presented with selected thickness. Figure 3(a) shows the cutoff changes of the UPS spectra, which reflect the vacuum-level change of the samples. It is very impressive that a very thin layer of $\mathrm{C}_{60}$ film can significantly change the vacuum level, thus the sample work function. The pristine perovskite film had a WF of $4.73 \mathrm{eV}$, which is consistent with the previous reports. ${ }^{29}$ With the $\mathrm{C}_{60}$ thickness increasing, the WF shifted to lower binding energy, which means higher WF. The WF was $6.15 \mathrm{eV}$ when the $\mathrm{C}_{60}$ thickness was at $2 \AA$ (data was not shown here). The change slowed down and finally saturated when the $\mathrm{C}_{60}$ thickness

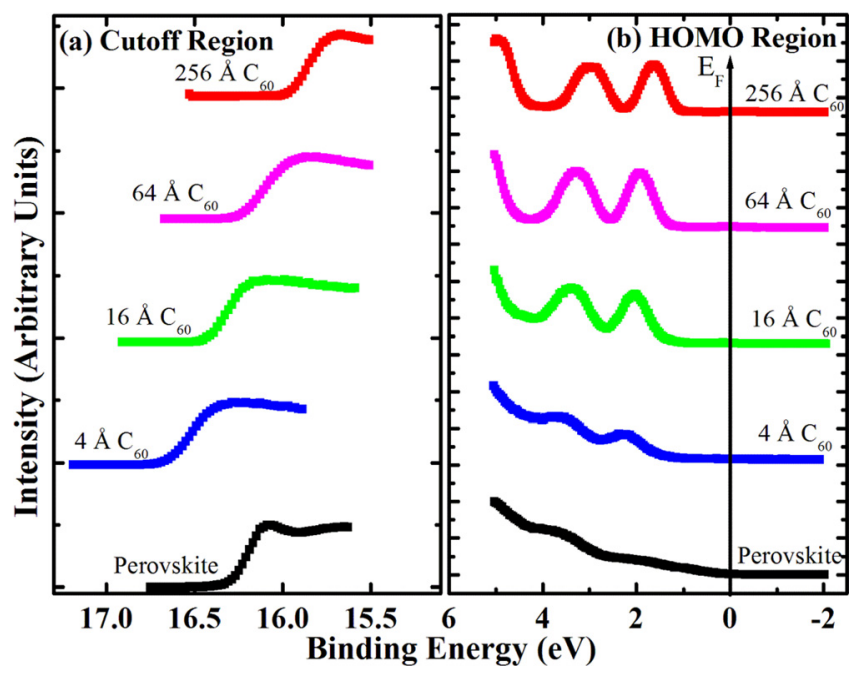

FIG. 3. (Color online) UPS data for $\mathrm{C}_{60}$ /perovskite: (a) the cut-off region and (b) the HOMO region.

reached to $128 \AA$. In Fig. 3(b), it shows the HOMO region of the UPS spectra of the same films. It can be observed that with the $\mathrm{C}_{60}$ thickness increasing, the HOMO shift followed the cutoff changes, implying that the $\mathrm{C}_{60}$ molecule does not undergo significant chemical modification. No new state emerges from the gap with the $\mathrm{C}_{60}$ thickness increasing. The shifts of the energy levels were due to the charge transfer from the perovskite films to $\mathrm{C}_{60}$ molecules.

To further reveal the thickness dependence and charge transfer process, we performed detailed XPS core level analysis (XPS data were not shown here). For C $1 \mathrm{~s}$, there was a strong initial shift to lower binding energy when $2 \AA \mathrm{C}_{60}$ deposited on the perovskite film. With the increasing $\mathrm{C}_{60}$ thickness, the shift trend to the lower binding energy slowed down and saturated when the thickness reached $128 \AA$. For all the spectra with the different $\mathrm{C}_{60}$ thickness, we observed the same initial strong shift to the lower binding energy at $2 \AA \mathrm{C}_{60}$. Then, the core levels shifted to higher binding energy at $4 \AA \mathrm{C}_{60}$. After the $\mathrm{C}_{60}$ thickness reached $8 \AA$, the core levels shifted to lower binding energy and finally saturated at $128 \AA \mathrm{C}_{60}$. We suspect that the strong initial shift to the lower binding energy for all spectra may due to the electron transfer from the perovskite to the $\mathrm{C}_{60}$ molecule. Further deposition of $\mathrm{C}_{60}$ makes solid $\mathrm{C}_{60}$, which results in a reduction of the electron transfer since there is no band bending anymore. The UPS results showed that with the $\mathrm{C}_{60}$ thickness increasing, the WF and HOMO levels shifted to the lower binding energy. Our XPS results have the same rigid shift trend, especially when the thickness is over $8 \AA$, as the interface charge transfer has completed and a rigid shift in the $\mathrm{C}_{60}$ is taking place.

The energy level alignment diagram at Au/perovskite interfaces is depicted in Fig. 4(a). The VL and CBM of pristine $\mathrm{CH}_{3} \mathrm{NH}_{3} \mathrm{PbI}_{3}$ film are 4.7 and $0.7 \mathrm{eV}$ above the $\mathrm{E}_{\mathrm{F}}$, respectively. The $\mathrm{VBM}$ is $1.0 \mathrm{eV}$ below the $\mathrm{E}_{\mathrm{F}}$. A gradual energy level shift is observed with the subsequent Au depositions. We neglected the intermediate charging processes because it was naturally eliminated without a significant impact on the final energy levels. On the side of perovskite, at the very interface of $\mathrm{Au} /$ perovskite, the VL, CBM, and VBM of $\mathrm{CH}_{3} \mathrm{NH}_{3} \mathrm{PbI}_{3}$ are $5.1,1.1 \mathrm{eV}$ above, and $0.6 \mathrm{eV}$

(a) Au/perovskite

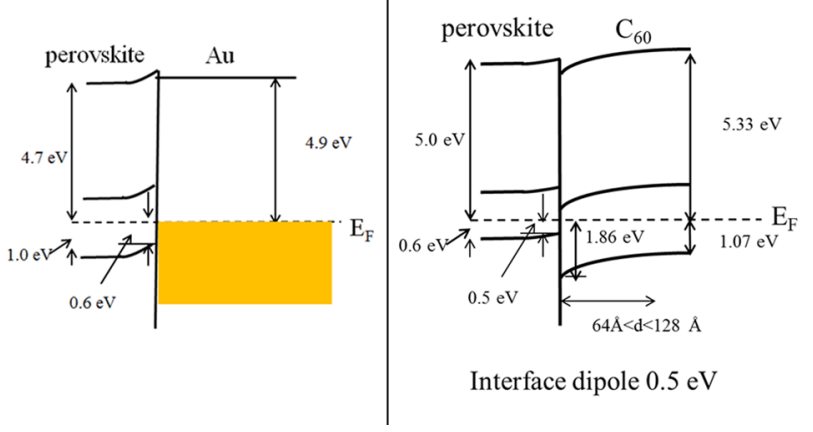

FIG. 4. (Color online) Energy level alignment diagram: (a) Au/perovskite and (b) $\mathrm{C}_{60}$ /perovskite. 
below the $\mathrm{E}_{\mathrm{F}}$, respectively. After considering the $0.4 \mathrm{eV}$ upward shift of energy levels with respect to the final deposition of $64 \AA \mathrm{Au}$, the VL is $5.2 \mathrm{eV}$ on the side of Au, indicating that there is an interface dipole of $0.1 \mathrm{eV}$ at the interface of $\mathrm{Au} /$ perovskite. The VBM of perovskite is brought to the $\mathrm{E}_{\mathrm{F}}$ of the system, resulting in a band bending, and thus, a built-in field in the perovskite film that encourages hole transport to the interface. The hole accumulation at the vicinity of the interface facilitates the hole transfer from $\mathrm{CH}_{3} \mathrm{NH}_{3} \mathrm{PbI}_{3}$ to $\mathrm{Au}$. Furthermore, the decreasing energy offset between the $\mathrm{VBM}$ of $\mathrm{CH}_{3} \mathrm{NH}_{3} \mathrm{PbI}_{3}$ and the $\mathrm{E}_{\mathrm{F}}$ indicates a decreasing energy loss for hole extraction from $\mathrm{CH}_{3} \mathrm{NH}_{3} \mathrm{PbI}_{3}$ to the Au. Given the favorable energy level alignment, the $\mathrm{Au} /$ perovskite interface can be effectively used as holes collector in perovskite-based solar cells.

The energy level diagram for $256 \AA \mathrm{C}_{60}$ /perovskite interface is depicted in Fig. 4(b). In Fig. 4(b), there is a $0.5 \mathrm{eV}$ interface dipole between perovskite and $\mathrm{C}_{60}$, and $\mathrm{HOMO}$ onset $\mathrm{BE}$ of perovskite was measured to be $0.6 \mathrm{eV}$. The WF of the perovskite was measured to be $5.0 \mathrm{eV}$. There is a clear band bending at the $\mathrm{C}_{60}$ region. The HOMO onset of $\mathrm{C}_{60}$ was measured to be $1.86 \mathrm{eV}$. With further $\mathrm{C}_{60}$ deposition, a gradual relaxation was observed, resulting in a band bending region of $\sim 200 \AA$. The energy level shift for perovskite $/ \mathrm{C}_{60}$ is due to the electron transfer from the perovskite to the $\mathrm{C}_{60}$. These are high energy level electrons that may have acted as charge recombination/scattering centers, and removing them from the perovskite should contribute to the reduction in trap states observed in the device studies. With the $\mathrm{C}_{60}$ thickness increasing, the electron transfer reduced and finally saturated when the $\mathrm{C}_{60}$ thickness reached to $128 \AA$. We also compared the $\mathrm{C}_{60}$ band structures with the previous report. ${ }^{30}$ We notice that the pristine $\mathrm{C}_{60}$ has the HOMO and LUMO levels at $1.38 \mathrm{eV}$ and $-0.92 \mathrm{eV}$, respectively. In this case, we discovered that the $\mathrm{C}_{60}$ was $\mathrm{n}$-type at the interface and became more neutral away from the interface as the HOMO level was pulled up and got closer to the Fermi level. Using thermal admittance spectroscopy (TAS), Shao et al. found that there was a relatively large density of defect states on the order of $1 \times 10^{17}$ to $1 \times 10^{19} \mathrm{~m}^{-3}$ in perovskite, producing large hysteresis of photocurrents. ${ }^{17}$ The trap density of state (tDOS) was reduced by one order of magnitude just after the spin coating of $\mathrm{C}_{60}$ on the perovskite films. Subsequent thermal annealing reduced the tDOS even more. The reduction may be the results of charge transfer at the interface that filled the trap states. It is possible that thermal annealing may drive $\mathrm{C}_{60}$ into the grain boundaries as individual molecules that act as strong electron absorbers as the $1 / 4$ monolayer case that further reduces the density of defect states.

\section{SUMMARY AND CONCLUSIONS}

In conclusion, we have measured the electronic structure evolution of $\mathrm{Au}$ on perovskite and $\mathrm{C}_{60}$ on perovskite. Au can efficiently lift the energy level of perovskite film by $0.4 \mathrm{eV}$, resulting in a band bending and thus a built-in field in perovskite. The enhanced hole accumulation at the interface due to the p-type self-doping behavior facilitates the hole transfer from perovskite to Au. The energy loss decreases significantly as hole extracting from perovskite to $\mathrm{Au}$ electrode due to the decreasing offset between the VBM of perovskite and the Fermi level of the system. Even though for an ntyped perovskite, it is confirmed that the Au/perovskite interface can be effectively used as holes collector in a perovskite-based solar cell, indicating a huge potential of Au/perovskite-based organometal trihalide perovskite planar structured solar cells. Furthermore, an initial charging of $0.3 \mathrm{eV}$ observed with the deposition of lower Au coverage can be attributed to Au clusters formed on the top of the perovskite film. The charging will be eliminated with thicker Au coverage of ca. $8 \AA$ following a metallic Fermi edge emergence. We observed that the WF of the perovskite is $5.0 \mathrm{eV}$ and the $\mathrm{VBM}$ is $0.6 \mathrm{eV}$. The band gap of the perovskite is $1.66 \mathrm{eV}$, which is in accordance with previous reports. There is an interface dipole as $0.5 \mathrm{eV}$ at the interface of perovskite and $\mathrm{C}_{60}$. We also observed the HOMO level of $\mathrm{C}_{60}$ shifts to lower binding energy, indicating a band bending in the $\mathrm{C}_{60}$ region. The perovskite core levels show a strong initial shift to lower binding energy, indicating electron transfer from the perovskite film to fullerene molecules. The strongest electron transfer happened at 1/4 monolayer of fullerene, and further deposition reduced the transfer as forms $\mathrm{C}_{60}$ solid film. We based our explanation on the energy level alignment from the photoemission spectroscopy, which indicates the clear charge transfer process between the perovskite and $\mathrm{C}_{60}$, and the results from the thermal admittance spectroscopy that show the removal of the trap states by $\mathrm{C}_{60}$.

\section{ACKNOWLEDGMENTS}

This work was supported in part by the National Science Foundation (Grant Nos. CBET-1437656 and DMR1303742) and the National Natural Science Foundation of China (Grant Nos. 51173205 and 11334014). X. L. Liu acknowledges support from National Natural Science Foundation of China (NSFC, 51203192, 11334014) and from the Natural Science Foundation of Hunan Province, China (Grant No. 12JJ3003). J. Huang thanks the financial supported from U.S. Department of Energy under Award No. DE-EE0006709 for the perovskite material preparation and data analysis.

${ }^{1}$ G. Hodes and D. Cahen, Nat. Photonics 8, 87 (2014).

${ }^{2}$ G. Hodes, Science 342, 317 (2013).

${ }^{3}$ H.-S. Kim et al., Sci. Rep. 2, 591 (2012).

${ }^{4}$ M. M. Lee, J. Teuscher, T. Miyasaka, T. N. Murakami, and H. J. Snaith, Science 338, 643 (2012).

${ }^{5}$ M. Liu, M. B. Johnston, and H. J. Snaith, Nature 501, 395 (2013).

${ }^{6}$ A. Abrusci, S. D. Stranks, P. Docampo, H.-L. Yip, A. K.-Y. Jen, and H. J. Snaith, Nano Lett. 13, 3124 (2013).

${ }^{7}$ S. D. Stranks, G. E. Eperon, G. Grancini, C. Menelaou, M. J. P. Alcocer, T. Leijtens, L. M. Herz, A. Petrozza, and H. J. Snaith, Science 342, 341 (2013).

${ }^{8}$ T. Baikie, Y. N. Fang, J. M. Kadro, M. Schreyer, F. X. Wei, S. G. Mhaisalkar, M. Graetzel, and T. J. White, J. Mater. Chem. A 1, 5628 (2013). 
${ }^{9}$ B. Conings, L. Baeten, C. De Dobbelaere, J. D'Haen, J. Manca, and H. G. Boyen, Adv. Mater. 26, 2041 (2014).

${ }^{10}$ C. Wehrenfennig, G. E. Eperon, M. B. Johnston, H. J. Snaith, and L. M. Herz, Adv. Mater. 26, 1584 (2014).

${ }^{11}$ G. Xing, N. Mathews, S. S. Lim, N. Yantara, X. Liu, D. Sabba, M. Grätzel, S. Mhaisalkar, and T. C. Sum, Nat. Mater. 13, 476 (2014).

${ }^{12}$ E. Edri, S. Kirmayer, S. Mukhopadhyay, K. Gartsman, G. Hodes, and D. Cahen, Nat. Commun. 5, 3461 (2014).

${ }^{13}$ Z. Xiao, C. Bi, Y. Shao, Q. Dong, Q. Wang, Y. Yuan, C. Wang, Y. Gao, and J. Huang, Energy Environ. Sci. 7, 2619 (2014).

${ }^{14}$ U. Bach, D. Lupo, P. Comte, J. E. Moser, F. Weissortel, J. Salbeck, H. Spreitzer, and M. Grätzel, Nature 395, 583 (1998).

${ }^{15}$ J. M. Ball, M. M. Lee, A. Hey, and H. J. Snaith, Energy Environ. Sci. 6, 1739 (2013).

${ }^{16} \mathrm{H}$. Zhou et al., Science 345, 542 (2014).

${ }^{17}$ Y. Shao, Z. Xiao, C. Bi, Y. Yuan, and J. Huang, Nat. Commun. 5, 5784 (2014).

${ }^{18}$ Q. Wang, Y. Shao, Q. Dong, Z. Xiao, Y. Yuan, and J. Huang, Energy Environ. Sci. 7, 2359 (2014).

${ }^{19}$ C. Bi, Y. Shao, Y. Yuan, Z. Xiao, C. Wang, Y. Gao, and J. Huang, J. Mater. Chem. A 2, 18508 (2014).
${ }^{20}$ A. Dualeh, N. Tétreault, T. Moehl, P. Gao, M. K. Nazeeruddin, and M. Grätzel, Adv. Funct. Mater. 24, 3250 (2014).

${ }^{21}$ Q. Chen, H. Zhou, Z. Hong, S. Luo, H. Duan, H. Wang, Y. Liu, G. Li, and Y. Yang, J. Am. Chem. Soc. 136, 622 (2014).

${ }^{22}$ G. Xing, N. Mathews, S. Sun, S. S. Lim, Y. M. Lam, M. Grätzel, S. Mhaisalkar, and T. C. Sum, Science 342, 344 (2013).

${ }^{23}$ H.-S. Kim, J.-W. Lee, N. Yantara, P. P. Boix, S. A. Kulkarni, S. Mhaisalkar, M. Grätzel, and N.-G. Park, Nano Lett. 13, 2412 (2013).

${ }^{24}$ Irfan, H. Ding, Y. Gao, C. Small, D. Y. Kim, J. Subbiah, and F. So, Appl. Phys. Lett. 96, 243307 (2010).

${ }^{25}$ Y. Gao, Mater. Sci. Eng., R 68, 39 (2010).

${ }^{26}$ A. C. Dürr et al., Phys. Rev. B 68, 115428 (2003).

${ }^{27}$ N. Koch, A. C. Dürr, J. Ghijsen, R. L. Johnson, J.-J. Pireaux, J. Schwartz, F. Schreiber, H. Dosch, and A. Kahn, Thin Solid Films 441, 145 (2003).

${ }^{28}$ H. Oji, E. Ito, M. Furuta, H. Ishii, Y. Ouchi, and K. Seki, Synth. Methods 121, 1721 (2001).

${ }^{29}$ A. Kojima, K. Teshima, Y. Shirai, and T. Miyasaka, J. Am. Chem. Soc. 131, 6050 (2009).

${ }^{30}$ M. Zhang, H. Ding, C. W. Tang, and Y. Gao, Org. Electron. 12, 1588 (2011). 